\title{
Propiedades ópticas y estructurales de las películas de óxido de silicio rico en silicio obtenidas por la técnica HFCVD
}

\section{Optical and structural properties of silicon rich oxide films obtained by HFCVD technique}

CASTILLO-TÉPOX, Nora†*, LUNA-LÓPEZ, José Alberto, HERNÁNDEZ-DE LA LUZ, José Álvaro David y MONFIL-LEYVA, Karim

Benemérita Universidad Autónoma de Puebla (BUAP), Centro de Investigación en Dispositivos Semiconductores (CIDSICUAP), Av. San Claudio y 14 sur, Edifs. IC5 y IC6 C.U., Col. Jardines de San Manuel, Puebla 72570, México.

ID $1^{\text {er }}$ Autor: Nora, Castillo-Tépox / ORC ID: 0000-0002-5582-8185, CVU CONACYT ID: 210633

ID $1^{\text {er }}$ Coautor: José Alberto, Luna-López /ORC ID: 0000-0002-7647-3184, CVU CONACYT ID: 200808

ID $2^{\text {do }}$ Coautor: José Álvaro David, Hernández-De la Luz /ORC ID: 0000-0002-7913-0240, CVU CONACYT ID: 240901

ID $3{ }^{\text {er }}$ Coautor: Karim, Monfil-Leyva /ORC ID: 0000-0002-4370-5655, CVU CONACYT ID: 37712

DOI: $10.35429 /$ JSL.2019.20.6.9.14

Recibido 23 Junio, 2019; Aceptado 20 Septiembre, 2019

\begin{abstract}
Resumen
En este trabajo se presentan resultados del análisis con relación al depósito y caracterización de películas de óxido de silicio rico en silicio (SRO). El depósito se realizó por la técnica de depósito químico en fase vapor asistido por filamento caliente (HFCVD), sobre sustratos de silicio tipo-p. El depósito se realizó variando la distancia de la fuente al sustrato (DFS) de 3, 4, 5 y $6 \mathrm{~mm}$. La cantidad de precursores de $\mathrm{SiO}_{2}$ fueron regulados considerando una distancia de $6 \mathrm{~mm}$ entre el filamento y la fuente (DFF) a una temperatura de $2000^{\circ} \mathrm{C}$ en el filamento. Se fijo un tiempo de depósito constante de 3 minutos y un nivel de flujo de hidrógeno de $10 \mathrm{sccm}$. La temperatura del sustrato fue de $450^{\circ} \mathrm{C}$ a $550^{\circ} \mathrm{C}$. Por perfilometría se obtiene el espesor de las películas que se encuentra en el rango de 200 a $600 \mathrm{~nm}$. Por medio de la espectroscopía infrarroja por transformada de Fourier (FTIR) se detectaron los modos vibracionales del SRO. La transmitancia más alta fue de $90 \%$ para las películas con DFS de $3 \mathrm{~mm}$. El band gap óptico varió de 2.2 a $3.3 \mathrm{eV}$. Al aplicarles un tratamiento térmico a la temperatura de $1000^{\circ} \mathrm{C}$ por una hora, las películas de SRO incrementaron su fotoluminiscencia en una orden de magnitud.
\end{abstract}

\begin{abstract}
In this work we present the results of the analysis obtained from the deposit and characterization of thin films of silicon rich oxide (SRO). The films were obtained by hot filament chemical vapor deposition (HFCVD) technique, such films were deposited on silicon substrates p-type. The deposit of thin films was realized considering different distances from source to substrate (DFS) which were 3, 4, 5 and $6 \mathrm{~mm}$. The quantity of precursors $\left(\mathrm{SiO}_{2}\right)$ was controlled by the distance from the filament to the source, which was $6 \mathrm{~mm}$ for this work, the filament was held at $2000^{\circ} \mathrm{C}$. A constant 3-minute deposit time was maintained, and the hydrogen flow level was $10 \mathrm{sccm}$. The films thickness was obtained by using the profilometry technique, the thickness range was from 200 to $600 \mathrm{~nm}$. The vibrational molecular modes of the SRO films were obtained by Fourier Transform Infrared Spectroscopy (FTIR). The films of $3 \mathrm{~mm}$ DFS exhibit an optical transmittance of $90 \%$. The optical energy band gap of the thin films varies from 2.2 to $3.3 \mathrm{eV}$. When an annealing process at $1000^{\circ} \mathrm{C}$ was carried out for one hour, the SRO films increase their photoluminescence by an order of magnitude approximately.
\end{abstract}

SRO, HFCVD, UV-Vis

SRO, HFCVD, UV-Vis

Citación: CASTILLO-TÉPOX, Nora, LUNA-LÓPEZ, José Alberto, HERNÁNDEZ-DE LA LUZ, José Álvaro David y MONFIL-LEYVA, Karim. Propiedades ópticas y estructurales de las películas de óxido de silicio rico en silicio obtenidas por la técnica HFCVD. Revista de Simulación y Laboratorio. 2019, 6-20: 9-14

*Correspondencia al Autor (Correo electrónico: noritacastillo@ homail.com)

$\dagger$ Investigador contribuyendo como primer Autor 


\section{Introducción}

El SRO es un material de dos fases el cual contiene nanocristales de silicio (nc-Si) en su microestructura atómica, tales nanocristales modifican sustancialmente las propiedades ópticas, estructurales y eléctricas de dicho material.

Las propiedades del SRO varían con el exceso de silicio contenido en las películas, convirtiéndolo en un material apto para ser utilizado en dispositivos optoelectrónicos. Las películas de SRO presentan un efecto interesante en el comportamiento de la corriente eléctrica cuando son iluminadas, siendo las nanopartículas de silicio y los defectos estructurales quienes provocan estos interesantes cambios (Tomozeiu, N., 2011, Carrier, P., Abramovici, G., Lewis, L. J., \& DharmaWardana, M. W. C., 2001).

Aunque las películas de SRO pueden ser depositadas por diferentes métodos, la técnica de depósito químico en fase vapor asistido por filamento caliente (HFCVD) ha reportado importantes características; tales como la facilidad en la variación de las condiciones de depósito de las películas. Estas condiciones de depósito son: la temperatura del sustrato (ts), el tiempo de depósito (td), la distancia del filamento a la fuente (dff), la distancia de la fuente al sustrato (dfs), el flujo de hidrógeno (fh) y las fuentes sólidas.

En el presente trabajo se reporta la influencia de la variación de la distancia fuente a sustrato, en las propiedades ópticas y estructurales de las películas de SRO.

\section{Metodología}

\section{Obtención de las películas de SRO}

El depósito de películas de SRO mediante la técnica HFCVD se realizó sobre obleas de silicio tipo P/Boro [100] de 2 pulgadas de diámetro con espesor de 254-304 $\mu \mathrm{m}$ y resistividad de 1-5 $\mathrm{ohm} / \mathrm{cm}$. Para la caracterización de la transmitancia se utilizaron sustratos de cuarzo. Los parámetros considerados para el depósito de las películas de SRO fueron los siguientes, td:3 minutos, fh: $10 \mathrm{sccm}$, dff: $6 \mathrm{~mm}$, dfs:3, 4, 5 y 6 $\mathrm{mm}$.
Para la obtención de los precursores volátiles se utilizaron 11 barras de cuarzo cilíndricas de $11 \mathrm{~cm}$ de largo y $2.5 \mathrm{~mm}$ de diámetro. La figura 1 muestra el esquema pictórico del sistema utilizado para el depósito de películas SRO mediante la técnica HFCVD.

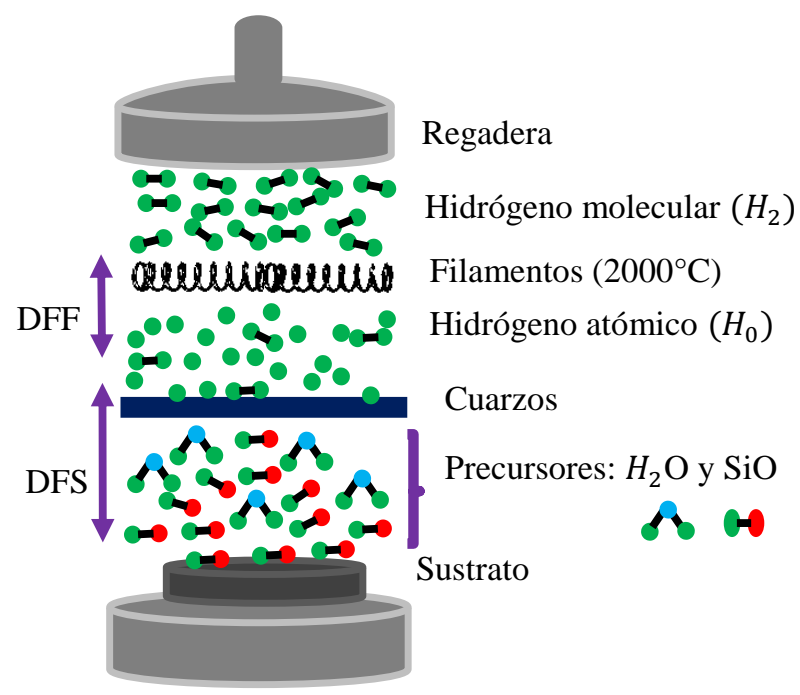

Figura 1 Sistema de depósito por la técnica HFCVD Fuente: Elaboración Propia

Los perfiles de temperatura del sustrato fueron de 450 a $550^{\circ} \mathrm{C}$ al momento de realizar el depósito.

A la mitad de cada muestra se le aplicó un tratamiento térmico (TT) por 1 hora a $1000^{\circ} \mathrm{C}$, esto se hizo con el objetivo de analizar las propiedades de las películas de SRO sin y con TT. En la tabla 1 presentamos el etiquetado de las muestras de SRO, indicando el nombre de cada muestra identificada, además se muestran las diferentes dfs.

\begin{tabular}{|} 
SRO: Flujo de hidrógeno de 10 sccm \\
\begin{tabular}{|l|l|l|l|l|}
\hline DFS & $3 \mathrm{~mm}$ & $4 \mathrm{~mm}$ & $5 \mathrm{~mm}$ & $6 \mathrm{~mm}$ \\
\hline Sin TT & A3 & A 4 & A5 & A6 \\
\hline Con TT & A3' & A4' & A5' & A6' \\
\hline
\end{tabular}
\end{tabular}

Tabla 1 Etiquetado de las películas de SRO Fuente: Elaboración Propia

\section{Caracterización de las películas SRO}

\section{Perfilometría}

Para determinar el espesor de las películas se consideraron 5 puntos por película, esto con el fin de lograr una medición más precisa de su espesor. Para esta caracterización se usó un perfilómetro Dektak 150 de superficie, con una resolución de $0.1 \mu \mathrm{m}$. 


\section{Espectroscopía infrarroja por transformadas de Fourier (FTIR)}

Para confirmar que las películas obtenidas son de SRO, se realizaron caracterizaciones para obtener los modos vibracionales característicos del SRO con un espectrofotómetro Bruker modelo Vector 22. Este espectrofotómetro es operado mediante el software OPUS NT versión 3 en un rango de 2000 a $400 \mathrm{~cm}^{-1}$ con una resolución de $1 \mathrm{~cm}^{-1}$.

\section{Espectroscopía UV-Vis}

Para obtener los espectros de transmitancia y estimar el ancho de banda del material se usó un espectrofotómetro UV-Visible Thermo Scientific Evolution 600, en un rango de longitudes de onda de 200 a $900 \mathrm{~nm}$ con una resolución de $0.5 \mathrm{~nm}$. Se utilizaron celdas de cuarzo de 1.4 milímetros de paso óptico.

\section{Fotoluminiscencia (FL)}

Para determinar la fotoluminiscencia del SRO se usó un espectroflurómetro FluroMax 3 de la marca Horiba Jobin Yvon, con una lámpara de excitación de xenón de 150 watts, una resolución de $0.3 \mathrm{~nm}$, en un rango de 370 a $1000 \mathrm{~nm}$ y un detector de emisión de alta sensibilidad, la longitud de onda de excitación utilizada fue de $335 \mathrm{~nm}$.

\section{Resultados}

\section{Espesor de las películas de SRO}

El análisis de la perfilometría de las películas se realizó con respecto de la variación de la DFS. En la gráfica 1, se observa un menor espesor en las películas con tratamiento térmico. Los mayores espesores se obtuvieron en las muestras depositadas con $4 \mathrm{~mm}$ de dfs.

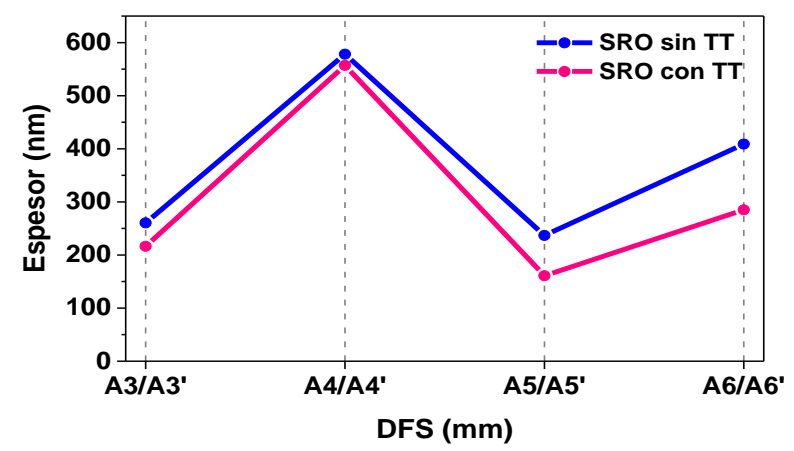

Gráfica 1 Espesores de las películas de SRO, con flujo de hidrógeno de $10 \mathrm{sccm}$

Fuente: Elaboración Propia

\section{Bandas de absorción del SRO}

En los espectros de FTIR obtenidos se identificaron las tres bandas de absorción característicos del óxido de silicio estequiométrico $\left(\mathrm{SiO}_{2}\right)$ con corrimientos en los picos correspondientes a los modos de balanceo en $458 \mathrm{~cm}^{-1}$, doblamiento en $812 \mathrm{~cm}^{-1} \mathrm{y}$ estiramiento en 1082 de los enlaces de $\mathrm{Si}-\mathrm{O}-\mathrm{Si}$, lo cual confirma que se obtuvieron películas de SRO (Luna-López, J. A., García-Salgado, G., Díaz-Becerril, T., López, J. C., VázquezValerdi, D. E., Juárez-Santiesteban, \& Coyopol, A. 2010). En la gráfica 2 se muestran los espectros de FTIR de las películas de SRO sin y con TT, se observa una mayor absorbancia en las muestras A4 y A4'.

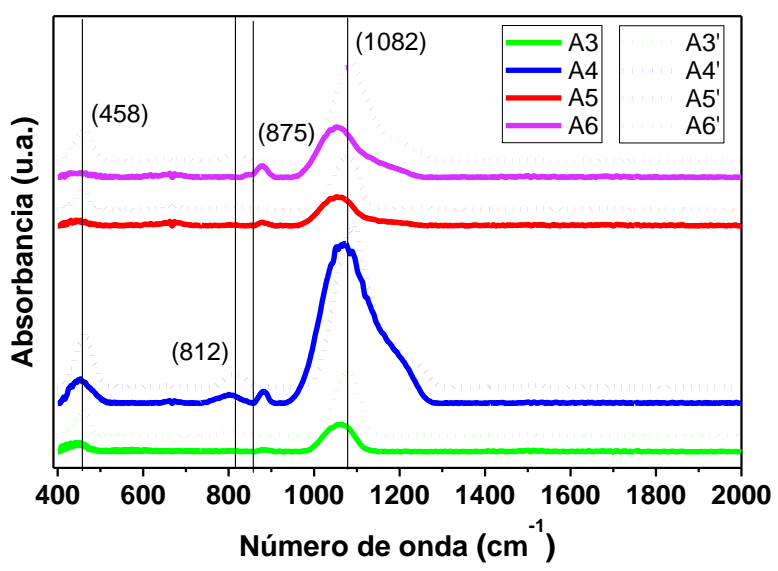

Gráfica 2 Espectros de FTIR de las películas de SRO sin y con TT

Fuente: Elaboración Propia

Los datos en la tabla 2 muestran un ligero corrimiento de las bandas hacia menores números de onda en balanceo y estiramiento de las películas de SRO sin TT, mientras que para las películas de SRO con TT, el corrimiento de las bandas en balanceo y estiramiento es hacia mayores longitudes de onda, tendiendo más a la estequiometría del $\mathrm{SiO}_{2}$. Debido al proceso de deshidrogenación se observa la ausencia del modo Si-H en el SRO con TT.

\begin{tabular}{|l|r|r|r|r|}
\hline & $\begin{array}{c}\text { Balanceo } \\
\text { Si-O-Si }\end{array}$ & \multicolumn{2}{|c|}{$\begin{array}{c}\text { Doblamiento } \\
\text { Si-O-Si }\end{array}$} & \multicolumn{2}{c|}{$\begin{array}{c}\text { Doblamiento } \\
\text { Si-H }\end{array}$} & $\begin{array}{c}\text { Estiramiento } \\
\text { Si-O-Si }\end{array}$ \\
\hline $\mathrm{SiO}_{2}$ & $\mathbf{4 5 8}$ & $\mathbf{8 1 2}$ & $\mathbf{8 7 5}$ & $\mathbf{1 0 8 2}$ \\
\hline $\mathrm{A} 3$ & 445 & - & 877 & 1051 \\
\hline $\mathrm{A} 3^{\prime}$ & $\mathbf{4 6 0}$ & $\mathbf{8 0 9}$ & - & $\mathbf{1 0 8 6}$ \\
\hline $\mathrm{A} 4$ & 447 & 800 & 878 & 1051 \\
\hline $\mathrm{A} 4{ }^{\prime}$ & $\mathbf{4 6 2}$ & $\mathbf{8 0 9}$ & - & $\mathbf{1 0 9 0}$ \\
\hline $\mathrm{A} 5$ & 447 & - & 877 & 1050 \\
\hline $\mathrm{A} 5$ & $\mathbf{4 6 0}$ & $\mathbf{8 1 0}$ & - & $\mathbf{1 0 8 0}$ \\
\hline $\mathrm{A} 6$ & 445 & - & 877 & 1050 \\
\hline A6 & $\mathbf{4 6 0}$ & $\mathbf{8 1 0}$ & - & $\mathbf{1 0 8 5}$ \\
\hline
\end{tabular}

Tabla 2 Modos vibracionales obtenidos mediante espectros de FTIR

Fuente: Elaboración Propia

CASTILLO-TÉPOX, Nora, LUNA-LÓPEZ, José Alberto, HERNÁNDEZ-DE LA LUZ, José Álvaro David y MONFIL-LEYVA, Karim. Propiedades ópticas y estructurales de las películas de óxido de silicio rico en silicio obtenidas por la técnica HFCVD. Revista de Simulación y Laboratorio. 2019 


\section{Transmitancia del SRO}

Los espectros de transmitancia muestran que las películas tienen una transmitancia aproximada del $65 \%$, excepto para las películas A3 y A3', que tienen una transmitancia arriba del $90 \%$. Las muestras A3 y A3' presentan un corrimiento en su borde de absorción hacia menores longitudes de onda. El tratamiento térmico aplicado a la muestra A3' cambio la estequiometría del material produciendo un mayor corrimiento hacia menores longitudes de onda, en la gráfica 3 se observan estos resultados.

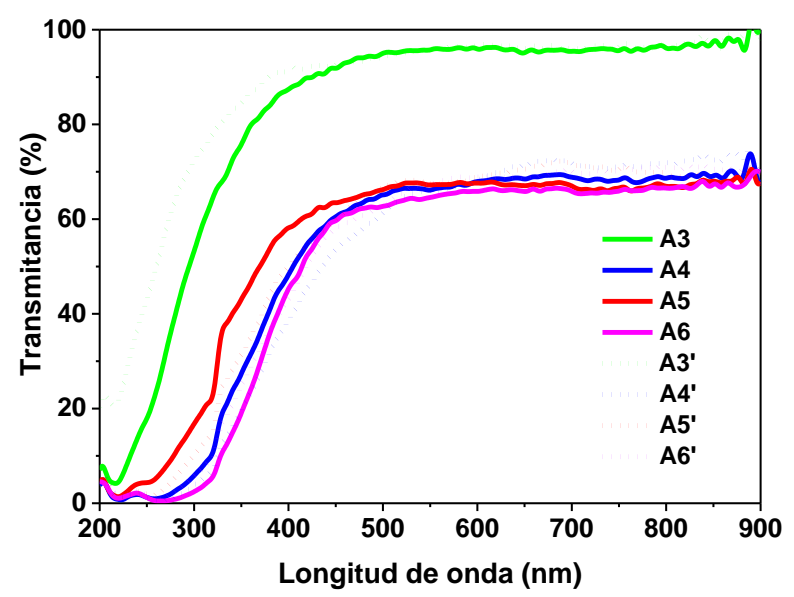

Gráfica 3 Transmitancia de las películas de SRO sin TT y con TT

Fuente: Elaboración Propia

\section{Coeficiente de absorción del SRO}

Mediante los espectros de transmitancia se determinó el coeficiente de absorción $(\alpha)$ y la banda de energía prohibida (Eg) de las películas de SRO. El cálculo del coeficiente de absorción se realizó utilizando la ley de Beer-Lambert (Donald, A. Neuman, 2003) dada por,

$I=I_{0} e^{(-\propto d)}$,

Donde, I, $\mathrm{I}_{0}$, son las intensidades de radiación saliente y entrante respectivamente (Donald, A. Neuman 2003). Se utilizaron los datos experimentales de la transmitancia y la perfilometría para calcular el coeficiente de absorción mediante la ecuación:

$\propto(h v)=\frac{-\ln [T(h v)]}{d}$,

Donde, $h v$ es la energía del fotón, $d$ el espesor, $T$ es la transmitancia y $\propto$ es el coeficiente de absorción. En la gráfica 4 se presentan los resultados del cálculo del coeficiente de absorción de las muestras de SRO, respecto a la dfs.

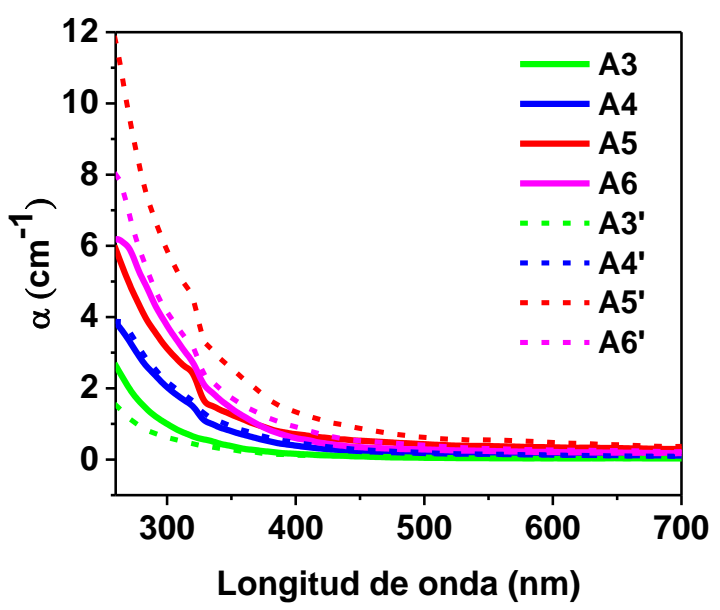

Gráfica 4 Coeficiente de absorción de las películas de SRO sin y con TT

Fuente: Elaboración Propia

\section{Valores estimados del band gap del SRO}

Lo valores aproximados de la banda de energía Eg se obtuvieron a partir de la relación (Donald, A. Neuman, 2003):

$$
\propto(h v)^{1 / n}=A(h v-E g),
$$

Conocida como gráfica de Tauc. Mediante la gráfica $(\alpha h v)^{1 / n} v s(h v)$ y la intersección de la pendiente de Tauc con el eje de la energía, se determinó el valor de $\mathrm{Eg}$, donde la mejor aproximación resulto ser $n=2$, ya reportado para este tipo de materiales. Un ejemplo de estos cálculos se muestra en la gráfica 5 .

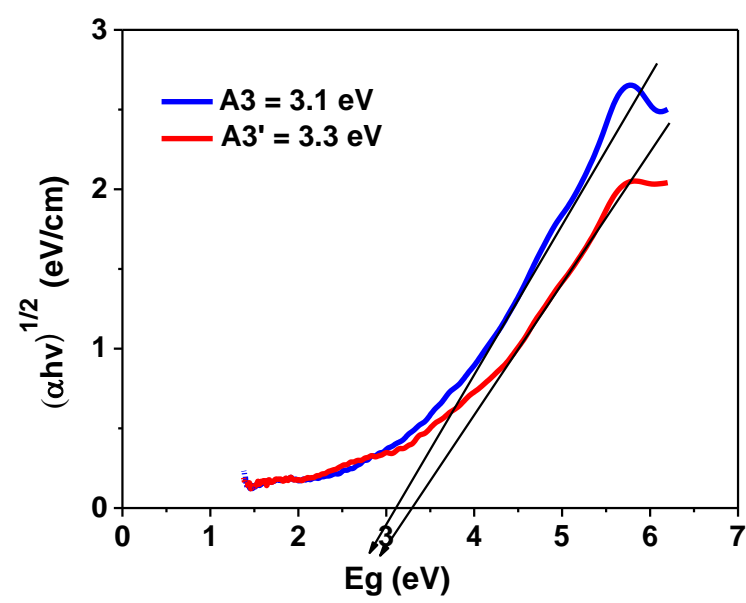

Gráfica 5 Cálculo de $E g$ de las películas de SRO sin y con TT usando el método de Tauc Plot Fuente: Elaboración Propia

En la tabla 3 se muestran los valores de $E g$ de las películas. 
Muestras $\mathrm{Eg}(\mathrm{eV})$

\begin{tabular}{|l|l|l|l|l|l|l|l|} 
A3 & A3' & A4 & A4' & A5 & A5' & A6 & A6' \\
\hline
\end{tabular}

\begin{tabular}{|l|l|l|l|l|l|l|l|}
\hline 3.1 & 3.3 & 2.5 & 2.4 & 2.3 & 2.4 & 2.5 & 2.4 \\
\hline
\end{tabular}

Tabla 3 Valores aproximados de $E g$ obtenidos de las muestras de SRO

Fuente: Elaboración Propia

\section{Fotoluminiscencia del SRO}

Con respecto a los espectros de FL, se puede observar en la gráfica 6 un aumento considerable tanto en la fotoluminiscencia de las películas con TT como en la intensidad de la FL en la banda correspondiente al cercano infrarrojo.

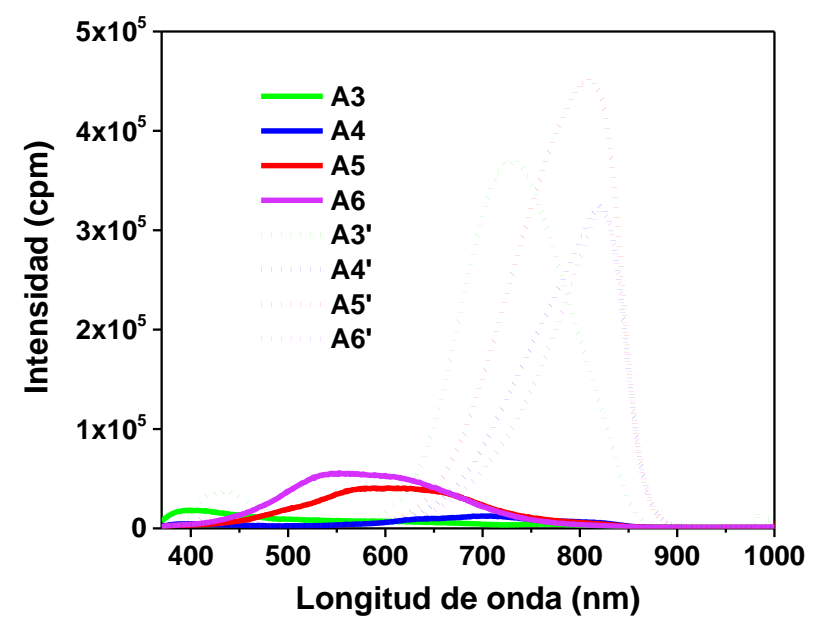

Gráfica 6 Espectros de fotoluminiscencia de las películas de SRO sin y con TT

Fuente: Elaboración Propia

Se ha reportado que para el SRO los mecanismos de emisión de luz visible e infrarrojo cercano se debe al efecto de confinamiento cuántico dimensional originado en los nanocristales de silicio (nc-Si) y a las transiciones radiativas generadas por los defectos en la matriz del óxido (Iacona, F., Franzo, G., \& Spinella, C. 2000. Inokuma, T., Wakayama, Y., Muramoto, T., Aoki, R., Kurata, Y., \& Hasegawa, S. 1998).

Se realizó una deconvolución de cada espectro encontrándose que la intensidad de los picos están relacionados con los siguientes tipos de defectos: enlace débil de oxígeno (WOB:415 $\mathrm{nm}$ ), vacancias neutrales de oxígeno (NOV:460 $\mathrm{nm})$, vacancias de oxígeno cargadas positivamente $\left(E^{\prime}{ }_{\delta}: 520 \mathrm{~nm}\right)$, centro hueco de oxígeno no enlazado (NBOHC:630 $\mathrm{nm}$ ), confinamiento cuántico (QC:760 nm), centros luminiscentes localizados en la interface de nc$\mathrm{Si} / \mathrm{SiO}_{2}$ (CLI:845 nm) (Ding, L., Chen, T. P., Liu, Y., Ng, C. Y., Yang, M., Wong, J. I., ... \& Huang, Y. 2008).

\section{Conclusiones}

En el presente trabajo se ha presentado la caracterización óptica de las películas de SRO depositadas por la técnica HFCVD para determinar sus efectos luminiscentes y de absorción considerando efectos térmicos. Además, se encontró que el espesor de las películas está relacionado con el decapado de las fuentes de cuarzo y es dependiente del flujo de $\mathrm{H}$. El espesor de las películas disminuye cuando son tratadas térmicamente, esto se debe a la reestructuración atómica del material cuando es sometido a altas temperaturas.

Los resultados de FTIR confirman que se obtuvieron películas de SRO. Se observa que las películas sin TT y con TT presentan una diferencia en la intensidad de las bandas características, teniendo mayor intensidad las películas con TT.

Las películas de menor espesor presentaron una alta transmitancia, aproximadamente del $90 \%$. El valor estimado de $E g$ se ubica en el rango de 2.3 a $3.3 \mathrm{eV}$, sin marcar ninguna tendencia en las dfs y al aplicarles el TT.

La fotoluminiscencia aumentó en una orden de magnitud al aplicarles el TT a las muestras, esto es atribuido a los efectos de confinamiento cuántico y a la activación de los defectos en la matriz del óxido.

Las propiedades ópticas de las películas de SRO pueden ser moduladas mediante la variación de la distancia entre fuente-sustrato lo que hace que ellas sean potencialmente útiles para construir dispositivos optoelectrónicos.

\section{Agradecimientos}

Los autores agradecen a los laboratorios de caracterización óptica, y estructural del Centro de investigación en Dispositivos Semiconductores (CIDS) del Instituto de Ciencias de la Benemérita Universidad Autónoma de Puebla (BUAP) y al laboratorio de Electrofotónica del Instituto Nacional de Astrofísica Óptica y Electrónica (INAOE). 


\section{Referencias}

A. Szekeres, E. Vlaikova, T. Lohner, P. Petrik, G. Huhn, K. Havancsak, I. Lisovskyy, S. Zlobi, I.Z. Indutnyy, \& P.E. Shepeliavyi (2009), Ellipsometric characterization of SiOx films with embedded $S i$ nanoparticles, Vacuum, 84, pp. 115-118.

Berman-Mendoza D., (2005). Sensor de radiación de silicio y SRO con eficiencia mejorada en el UV, Tesis Doctoral, INAOE, México.

Carrier, P., Abramovici, G., Lewis, L. J., \& Dharma-Wardana, M. W. C. (2001). Electronic and Optical Properties of Si/SiO 2 Superlattices from First Principles: Role of Interfaces. MRS Online Proceedings Library Archive, 677.

Coyopol, M. A. Cardona, T. Becerril, L. Licea Donald, A. Neamen, (2003), Semiconductors Physics and Devices. New York, NY: Mc Graw Hill.

Hernández-Simón Z. J. (2016) Propiedades Fotovoltaicas de Películas SRO depositadas mediante HFCVD en Estructuras ITO/SRO/Si y AU/SRO/Si. Tesis de maestría, CIDS BUAP.

Iacona, F., Franzó, G. \& Spinella, (2000) Correlation between luminescence and structural properties of Si nanocrystals, J. Appl. $\begin{array}{llll}\text { Phys., } & 87 & \text { (3), } & 1295 .\end{array}$ https://doi.org/10.1063/1.372013

Jiménez, \& A. M. Sánchez (2016), Silicon excess and thermal annealing effects on structural and optical properties of co-sputtered SRO films. J. Lumin., 176 pp. 40-46.

John, P. Mckelvey, (1984), Solid State and Semiconductor Physics. Malabar, Florida (USA): Krieger Publishing Company.

Luna-López JA, Vázquez-Valerdi D. E., Benítez-Lara A, García-Salgado G, Hernándezde la Luz AD, Morales Sánchez A, Flores Gracia FJ, \& Domínguez MA (2017) Optical and compositional properties of SiOx films deposited by HFCVD: effect of the hydrogen flow. J Elec Mater 2322. 46(4):2309$5271-1$
Luna-López, J. A., Carrillo-López, J., VázquezValerdi, D. E., García Salgado, G., DíazBecerril, T., Ponce Pedraza, A. \& Flores-Gracia, F. J. (2012) Morphological, compositional, structural, and optical properties of Si-nc embedded in SiOx films. Nanoscale Res Lett 7:604, $1-10$, 7:604. https://doi.org/10.1186/1556-276X-7604.

Luna-López, J. A., García-Salgado, G., DíazBecerril, T., López, J. C., Vázquez-Valerdi, D. E., Juárez-Santiesteban, \& Coyopol, A. (2010). FTIR, AFM and PL properties of thin SiOx films deposited by HFCVD. Materials Science and Engineering: B, 174(1-3), 88-92

Luna-López, J. A., Morales-Sánchez, A., Aceves Mijares, M., Yu, Z. \& Domínguez, C. (2008), Analysis of surface roughness and its relationship with photoluminescence properties of silicon-rich oxide films. Journal of Vacuum Science \& Technology A Vacuum Surfaces and Films. DOI: 10.1116/1.3032915.

Tomozeiu, N. (2011). Silicon Oxide (SiOx, O\&ltx\&lt2): a Challenging Material for Optoelectronics. In OptoelectronicsMaterials and Techniques. InTech.

Van Hapert, J. J., Vredenberg, A. M., Van Faassen, E. E., Tomozeiu, N., Arnoldbik, W. M., \& Habraken, F. H. P. M. (2004). Role of spinodal decomposition in the structure of SiOx. Physical Review B, 69(24), 245202.

Vázquez-Valerdi D., Luna-López J. A., CarrilloLópez J., García-Salgado G., Benítez-Lara A. \& Espinosa-Torres N., (2014) Compositional and optical properties of $\mathrm{SiO} x$ films and (SiOx/SiOy) junctions deposited by HFCVD. Nanoscale Research Letters. Vol. 422. https://doi.org/10.1186/1556-276X-9-422. 